\title{
Atomic Properties of the Two-Electron System using Variational Monte Carlo Technique
}

\author{
S.B. DOMA ${ }^{a, *, * *}$ AND F.N. EL-GAMMAL ${ }^{b}$
}

${ }^{a}$ Mathematics Department, Faculty of Science, Alexandria University, Alexandria, Egypt

${ }^{b}$ Mathematics Department, Faculty of Science, Menofia University, Shebin El-Kom, Egypt

(Received September 25, 2011)

\begin{abstract}
Using variational Monte Carlo method we calculated the lowest order relativistic corrections for the ground state energies of the helium-like atoms, up to $Z=10$, and also for some excited state energies of the helium atom. These relativistic corrections include: mass-velocity effect, orbit-orbit interaction, spin magnetic and dipole moments of the two electrons and the Darwin effect. Moreover, correction due to the nucleus motion has been also calculated. Our results were obtained by using two new types of compact and accurate trial wave functions for the helium ions. For excited states we used simple trial wave functions of good quality and accurate results. The obtained results are in good agreement with the most recent previous accurate values and also with the exact values.
\end{abstract}

PACS: 02.70.-c, 31.15.-p, 31.15.B-

\section{Introduction}

The nonrelativistic energies for the ground and excited states of helium and helium-like atoms are obtained by solving the nonrelativistic Schrödinger equation using many types of wave functions [1-8]. However, most of these studies are mainly based on the precision and convergence of variational wave functions instead of correction to the Hamiltonian, and hence the results obtained cannot be compared with experimental values. In order to compare theoretical atomic energies with their experimental counterparts, the nonrelativistic Schrödinger equation must be evaluated to high accuracy and a number of corrections such that the motion of the nucleus, radiative and quantum electrodynamics (QED) effects, must also be taken into account.

Many studies have been presented to calculate the relativistic corrections (both lowest and higher order), the mass related corrections, and some QED corrections for helium [9-11] and lithium [12-14] to an accuracy that meets or exceeds the current level of experimental precision. In 1958, Pekeris [15] presented accurate calculations for the relativistic corrections of two electron atoms with nuclear charge up to $Z=10$ using first-order perturbation theory and a wave function with many parameters. Also, variational and diffusion Monte Carlo techniques are used to calculate relativistic corrections to the ground state energies of $\mathrm{He}, \mathrm{Be}^{2+}, \mathrm{Ne}^{8+}, \mathrm{Be}$ and Ne atoms using an accurate non-relativistic wave function and first order perturbation theory [16]. In Ref. [17] the motion of the nucleus is taken into consideration to obtain the ground state energy of the helium atom and it was proven that the result of variational calculation would be lower than the experimental value when the nu-

\footnotetext{
* corresponding author; e-mail: sbdoma@yahoo.com

** Present address: Faculty of Information Technology and Computer Science, Sinai University, El-Arish, North Sinai, Egypt.
}

clear motion is ignored. Furthermore, they could prove that taking into account the Breit $\alpha^{2}$ (where $\alpha=1 / c$ is the fine structure constant) correction and correction of order $\alpha^{3}$ will improve the accuracy of the ground state energy to approach the corresponding experimental value. Stanke et al. [18] presented an approach to calculate the leading-order relativistic corrections for ground and excited states of helium isotopomers: ${ }^{3} \mathrm{He}$ and ${ }^{4} \mathrm{He}$. These corrections include the mass-velocity and Darwin terms, as well as terms due to magnetic orbit-orbit and Fermi contact interactions.

Recently, Alexander et al. [19] have proved that variational Monte Carlo (VMC) techniques can accurately evaluate the lowest-order relativistic corrections and the mass polarization term for the three lowest states of the helium atom with symmetry ${ }^{1} S,{ }^{1} P,{ }^{1} D,{ }^{3} S,{ }^{3} P$, and ${ }^{3} D$, using a set of medium-quality, explicitly correlated wave functions. The obtained results are in good agreement with that obtained using a high-quality Hylleraas wave function. The corrections of higher order in $\alpha$ including: $\alpha^{4}, \alpha^{5}$ and $\alpha^{6}$ have been calculated in many works [20-22]. These works demonstrate that these corrections are needed to achieve a high level of agreement between the experimental and the theoretical results. In our previous work [23] we used VMC method to calculate both the ground and excited states of helium atom and succeeded to obtain accurate results.

Extending from our previous work [23] and the results obtained in $[17,19]$, our goal in the present paper is to use VMC method to compute the lowest order relativistic corrections as well as the effect of the nucleus motion to the ground state of $\mathrm{He}$ atom and He-like isoelectronic ions up to $Z=10$ and also, for some excited states of helium atom.

\section{Method of calculations}

The VMC method is based on a combination of two ideas, namely the variational principal and Monte Carlo evaluation of integrals using importance sampling based on the Metropolis algorithm. It is used to compute quan- 
tum expectation values of an operator. In particular, if the operator is the Hamiltonian, its expectation value is the variational energy,

$$
E_{\mathrm{VMC}}=\frac{\int \Psi_{\mathrm{T}}^{*}(\boldsymbol{R}) \hat{H} \Psi_{\mathrm{T}}(\boldsymbol{R}) \mathrm{d} \boldsymbol{R}}{\int \Psi_{\mathrm{T}}^{*}(\boldsymbol{R}) \Psi_{\mathrm{T}}(\boldsymbol{R}) \mathrm{d} \boldsymbol{R}},
$$

where $\Psi_{\mathrm{T}}$ is a trial wave function and $\boldsymbol{R}$ is the $3 N$ -dimensional vector of the electron coordinates. According to the variational principle, a trial wave function for a given state must produce an energy which is above the exact value of that state; i.e. $E_{\mathrm{VMC}} \geq E_{\text {exact }}$.

To evaluate the integrals in Eq. (2.1) we firstly construct a trial wave function, $\Psi_{\mathrm{T}}^{\alpha}(\boldsymbol{R})$, depending on a set of $\alpha$-variational parameters $\alpha=\left(\alpha_{1} \alpha_{2}, \ldots \alpha_{N}\right)$ and then vary the parameters to obtain minimum energy.

Variational Monte Carlo calculations determine $E_{\mathrm{VMC}}$ by writing it as [24]:

$$
E_{\mathrm{VMC}}=\int P(\boldsymbol{R}) E_{L}(\boldsymbol{R}) \mathrm{d}(\boldsymbol{R})
$$

where $P(\boldsymbol{R})=\frac{\left|\Psi_{\mathrm{T}}(\boldsymbol{R})\right|^{2}}{\int\left|\Psi_{\mathrm{T}}(\boldsymbol{R})\right|^{2} \mathrm{~d} \boldsymbol{R}}$ is positive everywhere and interpreted as a probability distribution and $E_{L}=$ $\frac{\hat{H} \Psi_{\mathrm{T}}(\boldsymbol{R})}{\Psi_{\mathrm{T}}(\boldsymbol{R})}$ is the local energy function.

The value of $E_{L}$ is evaluated using a series of points $R_{i j}$ sampled from the probability density $P(\boldsymbol{R})$. At each of these points the weighted average $\left\langle E_{L}\right\rangle=\frac{\int \Psi_{T}^{2}(\boldsymbol{R}) E_{L} \mathrm{~d} \boldsymbol{R}}{\int \Psi_{\mathrm{T}}^{2}(\boldsymbol{R}) \mathrm{d} \boldsymbol{R}}$, is evaluated. After a sufficient number of evaluations the VMC estimate of $E_{\mathrm{VMC}}$ will be

$$
E_{\mathrm{VMC}}=\left\langle E_{L}\right\rangle=\lim _{N \rightarrow \infty} \lim _{L \rightarrow \infty} \frac{1}{N} \frac{1}{L} \sum_{j=1}^{N} \sum_{i=1}^{L} E_{L}\left(\boldsymbol{R}_{i j}\right),
$$

where $L$ is the ensemble size of random numbers $\left\{R_{1}, R_{2}, \ldots, R_{L}\right\}$, which may be generated using a variety of methods $[25,26]$ and $N$ is the number of ensembles. These ensembles so generated must reflect the distribution function itself. A given ensemble is chosen according to the Metropolis algorithm [27].

\section{Relativistic Hamiltonian for the two-particle systems}

The Hamiltonian of the two-electron atom which takes into account relativistic corrections (in atomic units (a.u.)) takes the form [28]:

$$
H=H_{0}+H_{1}+H_{2}+H_{3}+H_{4}+H_{5},
$$

where the description of each term is as follows:

(i) The first term $H_{0}$ represents the non-relativistic approximation and is given by

$$
H_{0}=\frac{1}{2}\left(p_{1}^{2}+p_{2}^{2}\right)-\frac{Z}{r_{1}}-\frac{Z}{r_{2}}+\frac{1}{r_{12}} .
$$

Here $r_{1}=\left|\boldsymbol{r}_{1}\right|$ and $r_{2}=\left|\boldsymbol{r}_{2}\right|$, where $\boldsymbol{r}_{1}$ and $\boldsymbol{r}_{2}$ denote the relative radius vectors of the two electrons with respect to the nucleus and $r_{12}$ is the distance between the two electrons, i.e. $r_{12}=\left|\boldsymbol{r}_{1}-\boldsymbol{r}_{2}\right|$.

(ii) The second term in (3.1) is given by

$$
H_{1}=\frac{-1}{M}\left(\nabla_{1} \cdot \nabla_{2}\right) \text {. }
$$

(iii) The third term in (3.1), $H_{2}$, is the polarization term and introduces the movement of the nucleus, where $M$ is the finite nucleus-to-electron mass ratio. The importance of this term appears especially when comparing with experimental values. The energies obtained by ignoring this term are always lower than the corresponding experimental values. Then we can say that taking into account the nucleus motion $\left(H_{1}\right)$ will raise the energy to approach experimental values. It is given by

$$
H_{2}=-\frac{1}{8 c^{2}}\left(p_{1}^{4}+p_{2}^{4}\right) \text {. }
$$

The term $\mathrm{H}_{2}$ is the mass-velocity correction and it is readily seen to be the relativistic effect due to the kinetic energy and it represents the dependence of the mass of the electron on the velocity.

(iv) The fourth term in (3.1), $H_{3}$, is defined by

$$
H_{3}=\frac{-1}{2 c^{2}}\left[\frac{\boldsymbol{p}_{1} \cdot \boldsymbol{p}_{2}}{r_{12}}+\frac{\left(\boldsymbol{r}_{12} \cdot \boldsymbol{p}_{1}\right)\left(\boldsymbol{r}_{12} \cdot \boldsymbol{p}_{2}\right)}{r_{12}^{3}}\right] \text {. }
$$

Now, $H_{3}$ is the orbit-orbit (retardation) term which represents the classical relativistic correction to the interaction between electrons.

(v) The fifth term in (3.1), $H_{4}$, is defined by

$$
H_{4}=\frac{\pi}{c^{2}}\left[Z \frac{\delta\left(r_{1}\right)+\delta\left(r_{2}\right)}{2}-\delta\left(r_{12}\right)\right] \text {. }
$$

The Hamiltonian $\mathrm{H}_{4}$ represents the electron-nucleus and electron-electron Darwin effect. The first term in $H_{4}$, the one-electron Darwin arises from the smearing of the electrons due to their high speed. It can be considered as a correction to the distance between the electrons and the nuclei resulting in an overall decrease in the Coulomb attraction. Consequently, this term always increases the total energy of the system. The second term is the two-electron Darwin correction term, as with the first term, it serves to correct point-like charge distributions. Since it is a two-electron term, it reduces the repulsion between electrons. The energy corresponding to this term is always negative because it is proportional to the minuscule probability of two electrons being at the same point in space; it is expected to be small. However, it is still important since it is closely related to the spinspin interaction term. Also, it allows an estimation of the two-electron Lamb-shift correction interaction term. The Darwin correction can be calculated either directly using the delta function or by applying the Poisson equations [29]:

$$
\nabla_{i}^{2} \frac{1}{r_{i}}=-4 \pi \delta\left(r_{i}\right), \quad \nabla_{i}^{2} \frac{1}{r_{i j}}=\nabla_{j}^{2} \frac{1}{r_{i j}}=-4 \pi \delta\left(r_{i j}\right) .
$$

Our calculations are based on using the Poisson equations to evaluate the Darwin correction. Then, we will have

$$
\begin{gathered}
H_{4}=\frac{-1}{8 c^{2}}\left[\left(\sum_{i=1,2} \nabla_{i}^{2}\right)\left(\sum_{j} \frac{Z}{r_{j}}\right)\right. \\
\left.-\left(\sum_{i=1,2} \nabla_{i}^{2}\right)\left(\sum_{i \neq j} \frac{1}{r_{i j}}\right)\right] .
\end{gathered}
$$


(vi) Finally; the sixth term in $(3.1), H_{5}$, is the spinspin correction term and is given by

$$
\begin{aligned}
H_{5} & =\frac{1}{c^{2}}\left[-\frac{8 \pi}{3}\left(\boldsymbol{S}_{1} \cdot \boldsymbol{S}_{2}\right) \delta\left(r_{12}\right)\right. \\
& \left.+\frac{1}{r_{12}^{3}}\left(\boldsymbol{S}_{1} \cdot \boldsymbol{S}_{2}-\frac{3\left(\boldsymbol{S}_{1} \cdot \boldsymbol{r}_{12}\right)\left(\boldsymbol{S}_{2} \cdot \boldsymbol{r}_{12}\right)}{r_{12}^{2}}\right)\right] .
\end{aligned}
$$

The physical significance of $H_{5}$ is to represent the interaction between the spin magnetic and dipole moments of the two electrons. For the ground state of helium-like atom the first term appearing in $H_{5}$ (the dipolar interaction term) is identically zero [19]. Furthermore, $H_{5}$ can be approximated by substituting: $\boldsymbol{S}_{1} \cdot \boldsymbol{S}_{2}=-3 / 4$, then we will have [17]:

$$
H_{5}=\frac{2 \pi}{c^{2}}\left[\delta\left(r_{12}\right)\right] \text {. }
$$

Replacing $\delta\left(r_{12}\right)$ by using the Poisson equations, $H_{5}$ takes the following form:

$$
H_{5}=\frac{-\pi}{4 c^{2}}\left\{\left(\sum_{i=1,2} \nabla_{i}^{2}\right)\left(\sum_{i \neq j} \frac{1}{r_{i j}}\right)\right\} .
$$

Now, we will indicate the method of calculating the expectation values of the corrections given by Eqs. (3.2) to (3.9). In our calculations we use the form of $H_{0}$ in the Hylleraas coordinates [30]:

$$
\begin{aligned}
H_{0} & =-\frac{1}{2}\left(\sum_{i=1}^{n} \frac{\partial^{2}}{\partial r_{i}^{2}}+\sum_{i=1}^{n} \frac{2}{r_{i}} \frac{\partial}{\partial r_{i}}+\sum_{i<j}^{n} 2 \frac{\partial^{2}}{\partial r_{i j}^{2}}\right. \\
& +\sum_{i<j}^{n} \frac{4}{r_{i j}} \frac{\partial}{\partial r_{i j}}+\sum_{i \neq j}^{n} \frac{r_{i}+r_{i j}-r_{j}}{r_{i} r_{i j}} \frac{\partial^{2}}{\partial r_{i} \partial r_{i j}} \\
& +\sum_{i \neq j}^{n} \sum_{k>j}^{n} \frac{r_{i j}+r_{i k}+r_{j k}}{r_{i j} r_{i k}} \frac{\partial^{2}}{\partial r_{i j} \partial r_{i k}} \\
& +\sum_{i=1}^{n} \frac{1}{r_{i}^{2}} \frac{\partial^{2}}{\partial \theta_{i}^{2}} \sum_{i=1}^{n} \frac{1}{r_{i}^{2} \sin ^{2} \theta_{i}} \frac{\partial^{2}}{\partial \varphi_{i}^{2}} \sum_{i=1}^{n} \frac{\cot \theta_{i}}{r_{i}^{2}} \frac{\partial}{\partial \theta_{i}} \\
& -\sum_{i \neq j}^{n}\left(\frac{r_{j} \cot \theta_{j}}{r_{i} r_{i j} \sin \theta_{i}}+\frac{1}{2} \cot \theta_{i} \frac{r_{i j}-r_{j}-r_{i}}{r_{i}^{2} r_{i j}}\right) \frac{\partial^{2}}{\partial \theta_{i} \partial r_{i j}} \\
& \left.-\sum_{i \neq j}^{n} \frac{r_{j} \cot \theta_{j}}{r_{i} r_{i j} \sin \theta_{i}} \sin \left(\varphi_{i}-\varphi_{j}\right) \frac{\partial^{2}}{\partial \varphi_{i} \partial r_{i j}}\right) \\
& +\sum_{i=1}^{n} \frac{-Z}{r_{i}}+\sum_{i<j}^{n} \frac{1}{r_{i j}} .
\end{aligned}
$$

For corrections given by Eqs. (3.3) to (3.8), we firstly identify the following quantity for any $3 N$-dimensional vector of electron coordinates $\boldsymbol{R}$ [16]:

$$
\boldsymbol{F}_{i}(\boldsymbol{R})=\Psi(\boldsymbol{R})^{-1} \nabla_{i} \Psi(\boldsymbol{R}), \quad i=1,2 .
$$

Then, in terms of $\boldsymbol{F}_{i}(\boldsymbol{R})$ we could calculate the various expectation values using the following formulae:

$$
\begin{aligned}
H_{1} & =\frac{1}{M} \sum_{i<j} \boldsymbol{F}_{i} \cdot \boldsymbol{F}_{j} \\
H_{2} & =\frac{-1}{8 c^{2}} \sum_{i}\left(\boldsymbol{\nabla}_{i} \cdot \boldsymbol{F}_{i}+\boldsymbol{F}_{i}^{2}\right)^{2}, \\
H_{3} & =\frac{-1}{2 c^{2}} \sum_{i<j}\left[\frac{\left(\boldsymbol{r}_{i j} \cdot \boldsymbol{F}_{i}\right)\left(\boldsymbol{r}_{i j} \cdot \boldsymbol{F}_{j}\right)}{r_{i j}^{3}}+\frac{\boldsymbol{F}_{i} \cdot \boldsymbol{F}_{j}}{r_{i j}}\right], \\
H_{4}= & \frac{1}{4 c^{2}}\left(\sum_{i}\left[\nabla_{i} \cdot \boldsymbol{F}_{i}+2 \boldsymbol{F}_{i}^{2}\right]\right) \\
\times & \left(\sum_{j} \frac{-Z}{r_{j}}+\sum_{j<k} \frac{1}{r_{j k}}\right), \\
H_{5}= & \frac{-1}{2 c^{2}}\left(\sum_{i<j}\left[\nabla_{i} \cdot \boldsymbol{F}_{i}+2 \boldsymbol{F}_{i}^{2}\right]\right)\left(\sum_{j<k} \frac{1}{r_{j k}}\right) .
\end{aligned}
$$

\section{Ground-state of the helium-like atom}

Our calculations for the ground-state of helium and its isoelectronic ions are based on using two different types of trial wave functions, the first one is a highly compact wave function that has a clear physical meaning and satisfies all the boundary conditions; this wave function is proposed firstly in [31] and is given by

$$
\begin{aligned}
& \Psi_{1}\left(r_{1}, r_{2}, r_{12}\right)=(1+\hat{\boldsymbol{P}}) \exp \left(\frac{a_{1} r_{1}+b_{1} r_{1}^{2}}{1+r_{1}}\right) \\
& \times \exp \left(\frac{a_{2} r_{2}+b_{2} r_{2}^{2}}{1+r_{2}}\right) \exp \left(\frac{d r_{12}}{1+e r_{12}}\right)
\end{aligned}
$$

where $a_{1}, a_{2}, b_{1}, b_{2}, d$, and $e$ are variational parameters and $\hat{\boldsymbol{P}}$ is the operator that permutes the two electrons. The new in this wave function is the functional form: $\exp \left(\frac{a r+b r^{2}}{1+r}\right)$ which helps in satisfying Kato-cusp conditions, which have been stressed in the construction of an accurate wave function in the past. This wave function was used to calculate the ground state energy for the $\mathrm{He}$ atom and He-like isoelectronic ions for $Z=1$ to 10 and the results obtained were better than previous works for compact wave functions for the two-electron systems. Here we discuss the validity of using this compact wave function for calculating the relativistic corrections given by Eq. (3.1) for the ground state energies of helium-like atoms $\{Z=1,2, \ldots, 10\}$. The second type of trial wave function takes the form

$$
\begin{aligned}
& \Psi_{2}\left(r_{1}, r_{2}, r_{12}\right)=\left(\frac{2 \beta+1-\mathrm{e}^{-\beta r_{12}}}{2 \beta}\right) \mathrm{e}^{-Z\left(r_{1}+r_{2}\right)} \\
& \times\left[1+C_{1}\left(r_{1}^{2}+r_{2}^{2}\right)+C_{2}\left(r_{1}^{4}+r_{2}^{4}\right)\right],
\end{aligned}
$$

where $\beta, C_{1}$ and $C_{2}$ are variational parameters. $\Psi_{2}$ has been constructed by [32] as a simple function having accuracies and shapes similar to those given by Green et al. [33] or by Chandrasekhar et al. [34, 35], but with correct cusp conditions. $\Psi_{2}$ is a product of hydrogenic one-electron solutions and a fully correlated part satisfies all 
TABLE I

Expectation values of the polarization term $H_{1}$ for helium isoelectronic ions and nuclear mass $M$ for $Z=$ $1,2, \ldots, 10$.

\begin{tabular}{|c|c|c|}
\hline$Z$ & $\begin{array}{c}\text { The polarization } \\
\text { term }\left\langle H_{1}\right\rangle\end{array}$ & $M$ \\
\hline 1 & $\begin{array}{l}0.00001797(8)^{a} \\
0.00001793(6)^{b} \\
0.0000178874^{c} \\
0.00001790703^{d}\end{array}$ & $1836.1526724^{c}$ \\
\hline 2 & $\begin{array}{l}0.00002183(4)^{a} \\
0.00002189(1)^{b} \\
0.0000218014^{c} \\
0.00002180505^{d}\end{array}$ & $7294.299536^{c}$ \\
\hline 3 & $\begin{array}{l}0.00002263(6)^{a} \\
0.00002298(2)^{b} \\
0.00002259672^{c} \\
0.0000226012^{d}\end{array}$ & $12786.393087^{c}$ \\
\hline 4 & $\begin{array}{l}0.00002569(1)^{a} \\
0.00002599(2)^{b} \\
0.000025600578^{c} \\
0.00002560361^{b}\end{array}$ & $16424.203212^{c}$ \\
\hline 5 & $\begin{array}{l}0.00002749(2)^{a} \\
0.00002949(6)^{b} \\
0.00002754709^{c} \\
0.0000275490^{d}\end{array}$ & $20063.736514^{c}$ \\
\hline 6 & $\begin{array}{l}0.00003138(2)^{a} \\
0.00003139(3)^{b} \\
0.000031335806^{c} \\
0.00003133991^{b}\end{array}$ & 21868.662136 \\
\hline 7 & $\begin{array}{l}0.00003210(1)^{a} \\
0.00003299(4)^{b} \\
0.000032056630^{d} \\
0.00003205963^{d}\end{array}$ & $25519.042727^{c}$ \\
\hline 8 & $\begin{array}{l}0.00003279(3)^{a} \\
0.00003269(6)^{b} \\
0.000032624295^{c} \\
0.00003262448^{b}\end{array}$ & $29148.946104^{c}$ \\
\hline 9 & $\begin{array}{l}0.00003185(9)^{a} \\
0.00003131(4)^{b} \\
0.0000313075833^{c} \\
0.00003130749^{d}\end{array}$ & $34622.970927^{c}$ \\
\hline 10 & $\begin{array}{l}0.00003349(2)^{a} \\
0.00003374(1)^{b} \\
0.0000334032008^{c} \\
0.00003340341^{d}\end{array}$ & $36433.989510^{c}$ \\
\hline
\end{tabular}

${ }^{a}$ Results obtained using three-parameter wave function $\Psi_{1}$

${ }^{b}$ Results obtained using trial wave function $\Psi_{2}$.

${ }^{c}$ Accurate results from [5].

${ }^{d}$ Exact results from [15].

the coalescence cusp conditions at the Coulomb singularities. The application of using $\Psi_{2}$ was extended not only for ground state of helium but also to other ions belonging to the He isoelectronic sequence up to $Z=10$. The results obtained are in good agreement with the exact values. Using the above two types of trial wave functions we have calculated the relativistic corrections given by Eq. (3.1) for $Z=\{1, \ldots, 10\}$. In Table I we present the expectation values of the polarization term $H_{1}$ for helium isoelectronic ions and nuclear mass $M$ for $Z=1,2, \ldots, 10$.

\section{Excited states of the helium atom}

In this section we present the trial wave functions for the lowest four excited states corresponding to the configurations $1 s 2 s$ and $1 s 2 p$.

1. For the lowest ortho (space-antisymmetric) state $2^{3} S$, corresponding to the configuration $1 s 2 s$, the wave function takes the form

$$
\begin{aligned}
& \Psi_{2^{3} S}\left(\boldsymbol{r}_{1}, \boldsymbol{r}_{2}\right)=N\left[u_{1 s}\left(\boldsymbol{r}_{1}\right) v_{2 s}\left(\boldsymbol{r}_{2}\right)-v_{2 s}\left(\boldsymbol{r}_{1}\right) u_{1 s}\left(\boldsymbol{r}_{2}\right)\right] \\
& \quad \times f_{1}\left(r_{12}\right),
\end{aligned}
$$

where

$$
\begin{aligned}
& u_{1 s}(\boldsymbol{r})=\exp \left(-Z_{i} r\right), \\
& v_{2 s}(\boldsymbol{r})=\left(1-Z_{0} r / 2\right) \exp \left(-z_{0} r / 2\right),
\end{aligned}
$$

and $Z_{i}, Z_{0}$ are variational parameters and $N$ is a normalization constant.

2. The $2{ }^{1} S$ state (space-symmetric) is para-state corresponding to the configuration $1 s 2 s$. We construct a simple wave function which would be space-symmetric and orthogonal to the ground state in the form

$$
\begin{aligned}
& \Psi_{2^{1} S}\left(\boldsymbol{r}_{1}, \boldsymbol{r}_{2}\right)=N\left[u_{1 s}\left(\boldsymbol{r}_{1}\right) v_{2 s}\left(\boldsymbol{r}_{2}\right)+v_{2 s}\left(\boldsymbol{r}_{1}\right) u_{1 s}\left(\boldsymbol{r}_{2}\right)\right] \\
& \quad \times f_{1}\left(r_{12}\right),
\end{aligned}
$$

where

$$
\begin{aligned}
& u_{1 s}(\boldsymbol{r})=\exp (-2 r), \\
& v_{2 s}(\boldsymbol{r})=\exp \left(-\tau_{1} r\right)-C r \exp \left(-\tau_{2} r\right),
\end{aligned}
$$

and $\tau_{1}, \tau_{2}$, and $C$ are variational parameters.

The above trial wave functions were firstly presented in [36] but without taking into account electron-electron correlation. In our work we modified these trial wave functions by adding the term $f_{1}\left(r_{12}\right)$ which expresses the correlation between the two electrons due to their Coulomb repulsion. That is, we expect $f$ to be small when $r_{12}$ is small and to approach a large constant value as the electrons become well separated. A convenient and reasonable choice for $f_{1}\left(r_{12}\right)$ is

$$
f_{1}\left(r_{12}\right)=\exp \left(\frac{r}{\alpha(1+\beta r)}\right),
$$

where $\alpha$ and $\beta$ are additional positive variational parameters. The variational parameter $\beta$ controls the distance over which the trial wave function heals to its uncorrelated value as the two electrons separate. Using the cusp conditions [37] we can easily verify that the variational parameter $\alpha$ is given by $\alpha=2$.

3 . For the $2^{1} P$-state, which are the lowest para-states corresponding to the configuration $1 s 2 p$, simple trial wave functions for these states may be taken as

$$
\begin{gathered}
\Psi_{2^{1} P}\left(\boldsymbol{r}_{1}, \boldsymbol{r}_{2}\right)=\left(\mathrm{e}^{-Z r_{1}} r_{2} \cos \theta_{2} \mathrm{e}^{-a r_{2}}\right. \\
\left.+\mathrm{e}^{-Z r_{2}} r_{1} \cos \theta_{1} \mathrm{e}^{-a r_{1}}\right) f_{2}\left(r_{12}\right),
\end{gathered}
$$

and 


$$
f_{2}\left(r_{12}\right)=1-\frac{1}{1+2 \lambda} \mathrm{e}^{-\lambda r_{12}}
$$

4. The $2^{3} P$-state is the lowest ortho-state, corresponding to the configuration $1 s 2 p$ Simple trial wave functions for this state take the form

$$
\begin{gathered}
\Psi_{2^{3} P}\left(\boldsymbol{r}_{1}, \boldsymbol{r}_{2}\right)=\left(\mathrm{e}^{-Z r_{1}} r_{2} \cos \theta_{2} \mathrm{e}^{-a r_{2}}\right. \\
\left.-\mathrm{e}^{-Z r_{2}} r_{1} \cos \theta_{1} \mathrm{e}^{-a r_{1}}\right) f_{3}\left(r_{12}\right),
\end{gathered}
$$

where $f_{3}\left(r_{12}\right)$ is now given by

$$
f_{3}\left(r_{12}\right)=1-\frac{1}{1+4 \lambda} \mathrm{e}^{-\lambda r_{12}}
$$

In the above equations $a$ and $\lambda$ are variational parameters. The wave functions $\Psi_{2^{1} P}$ and $\Psi_{2^{3} P}$ were introduced by Christophe Pain [38]. It has been proven that using variational method, these wave functions provide quite accurate values for the energies and compact expressions for the wave functions particularly in the asymptotic region, which is important in the description of inter-particle interaction. In our work we have used $\Psi_{2^{3} S}$, $\Psi_{2^{1} S}, \Psi_{2^{1} P}$ and $\Psi_{2^{3} P}$ together with VMC method to obtain both non-relativistic energies and relativistic corrections for the lowest four excited states of the helium atom.

\section{Results}

The Monte Carlo method described here has been employed for calculating the Breit $\alpha^{2}$ corrections and the correction due to nucleus movement for the ground state energy of helium atom and its isoelectronic ions. All energies are obtained in atomic units i.e. $\left(\hbar=e=m_{\mathrm{e}}=1\right)$ with $c=137.035999679$ and a set of $4 \times 10^{7}$ Monte Carlo integration points in order to make the statistical error as low as possible. The values of the nuclear mass $M$ in Eq. (3.3) for different values of $Z=1,2, \ldots, 10$ which are presented in Table I are taken from [5]. The parameters appearing in $\Psi_{1}$ were optimized in Ref. [31] and it has been proven that these parameters can be written in a general form depending on the nuclear charge $Z$. Firstly, the two parameters $a_{1}, a_{2}$ and $d$ were fixed $\left(a_{1}=a_{2}=\right.$ $-Z, d=0.5)$ in order to satisfy all the cusp conditions. Then, other parameters are given by the following relations: $b_{1}=-1.0778 \times Z, b_{2}=0.4142-0.8287 \times Z$, and $e=0.2247 \times Z$. Then, the wave function $\Psi_{1}$ exhibits the advantage that it does not need to be optimized each time for a given $Z$ value. In our calculations we take the values of the variational parameters in $\Psi_{2}$ from Ref. [32]. In Table I we introduced the values of the expectation values of the polarization term up to $Z=10$ which represent the effect of the nucleus motion. From Table I, we can see that our results have a good accuracy that can be compared with both the most recent results and the exact values. Also, it is clear that by adding this correction, the energies will be raised. This concept was proven for the ground state $(Z=2)$ of helium atom in [17]. Then, we could now generalize it to include all helium ions up to $Z=10$. In Table II we displayed our results obtained for non-relativistic energies and the relativistic corrections using the trial wave functions $\Psi_{1}$ and $\Psi_{2}$. The results presented in Ref. [16] using VMC method for $Z=2$, 4, 10 are introduced for comparison together with the exact Pekeris values [15]. It is clear from Table II that our results are in most cases better than those obtained using variational Monte Carlo in [16]. In general, the results are in good agreement with the corresponding exact data. Figure 1a represents $\Delta E$ versus the nuclear charge $Z$, where $\Delta E=E_{\text {exact }}-E_{0}$, i.e. the difference between the non-relativistic energies corresponding to the expectation value of $H_{0}$ and the corresponding exact values using both $\Psi_{1}$ and $\Psi_{2}$.
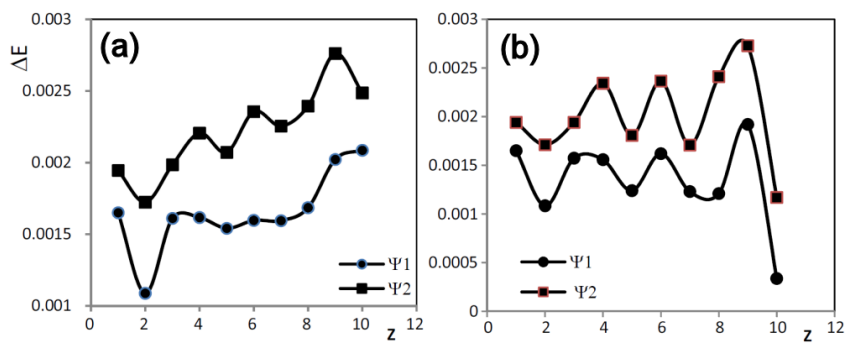

Fig. 1. (a) Difference between the calculated nonrelativistic energies and corresponding exact data, $\Delta E=$ $E_{\text {exact }}-E_{0}$, versus the nuclear charge $Z$. (b) Difference between the obtained relativistic energies and relativistic exact energies, $\Delta E=E_{\text {rel-exact }}-E_{\text {rel }}$, against the nuclear charge $Z$.

Accordingly, Fig. 1a displays a graphical representation of the comparison between the calculated values of the nonrelativistic energies by using the two-wave functions. This comparison shows that the results obtained using $\Psi_{1}$ are much better than those obtained by $\Psi_{2}$. In Fig. 1b, $\Delta E$ represents the difference between the total relativistic energies and the total exact energies, i.e. $\Delta E=E_{\text {rel-exact }}-E_{\text {rel }}$. The total relativistic energies are defined as $E_{\mathrm{rel}}=E_{0}+E_{1}+E_{2}+E_{3}+E_{4}+E_{5}\left(E_{i}=\left\langle H_{i}\right\rangle\right)$, while the total exact energies are defined as the sum of the exact nonrelativistic energies and the exact values of the relativistic and nucleus movement corrections. Figure $1 \mathrm{~b}$ confirms that the results obtained using $\Psi_{1}$ are more accurate.

For excited states of helium, all the values of the variational parameters appearing in the trial wave functions were optimized to minimize the energy. For the excited states of helium we increased the VMC integration points from $4 \times 10^{7}$ to $7 \times 10^{7}$ in order to achieve more good and accurate results with our choice of trial wave functions. In this way our results approach the corresponding values obtained in [19]. Table III displays the nonrelativistic energies and the relativistic corrections for the lowest four excited states of helium atom. We also introduced the most recent results obtained in previous works using VMC method but with different forms of trial wave functions [19]. 
TABLE II

Relativistic correction of helium and helium isoelectronic using wave function $\Psi_{1}$ and $\Psi_{2}$, the values in the parentheses are statistical errors.

\begin{tabular}{|c|c|c|c|c|c|}
\hline$Z$ & $\begin{array}{c}\text { Nonrelativistic } \\
\text { energies }\end{array}$ & $H_{2}$ & $H_{3}$ & $H_{4}$ & $H_{5}$ \\
\hline 1 & $\begin{array}{l}-0.5261001(9)^{a} \\
-0.5258080(6)^{b} \\
-0.527750974^{c}\end{array}$ & $\begin{array}{l}-0.00003287(2)^{a} \\
-0.00003276(2)^{b} \\
-0.000032776^{c}\end{array}$ & $\begin{array}{l}-0.000000460(2)^{a} \\
-0.000000473(4)^{b} \\
-0.000000472606^{c}\end{array}$ & $\begin{array}{l}0.00002650(2)^{a} \\
0.00002600(2)^{b} \\
0.00002707205^{c}\end{array}$ & $\begin{array}{l}0.000000723313(5)^{a} \\
0.000001071133(9)^{b} \\
0.0000009178099^{c}\end{array}$ \\
\hline 2 & $\begin{array}{l}-2.902637(3)^{a} \\
-2.902011(3)^{b} \\
-2.903724311^{c} \\
-2.89933(1)^{d}\end{array}$ & $\begin{array}{l}-0.00072001(2)^{a} \\
-0.00072218(7)^{b} \\
-0.000720074^{c} \\
-0.000712^{d}\end{array}$ & $\begin{array}{l}-0.000007670(1)^{a} \\
-0.000007396(2)^{b} \\
-0.000007407530^{c} \\
-0.000008188^{d}\end{array}$ & $\begin{array}{l}0.00058803(4)^{a} \\
0.00058801(1)^{b} \\
0.00058818411^{c} \\
0.0005838^{d}\end{array}$ & $\begin{array}{l}0.000033522775(1)^{a} \\
0.000036414962(4)^{b} \\
0.00003561617956^{c} \\
0.0000384^{d}\end{array}$ \\
\hline 3 & $\begin{array}{l}-7.278302(3)^{a} \\
-7.277929(3)^{b} \\
-7.279913341^{c}\end{array}$ & $\begin{array}{l}-0.00410013(2)^{a} \\
-0.00410122(2)^{b} \\
-0.004134249^{c}\end{array}$ & $\begin{array}{l}-0.000022880(2)^{a} \\
-0.000022585(1)^{b} \\
-0.00002279264^{c}\end{array}$ & $\begin{array}{l}0.00328106(3)^{a} \\
0.00327477(3)^{b} \\
0.00335086276^{c}\end{array}$ & $\begin{array}{l}0.000176552353(2)^{a} \\
0.000178705591(3)^{b} \\
0.00017870582527^{c}\end{array}$ \\
\hline 4 & $\begin{array}{l}-13.65395(3)^{a} \\
-13.65336(3)^{b} \\
-13.65556617^{c} \\
-13.65163(3)^{d}\end{array}$ & $\begin{array}{l}-0.01393454(7)^{a} \\
-0.01379164(4)^{b} \\
-0.013942403^{c} \\
-0.01389^{d}\end{array}$ & $\begin{array}{l}-0.000050840(2)^{a} \\
-0.000047853(1)^{b} \\
-0.00004679729^{c} \\
-0.0000488^{d}\end{array}$ & $\begin{array}{l}0.01120014(1)^{a} \\
0.01124437(1)^{b} \\
0.011258229^{c} \\
0.0112067^{d}\end{array}$ & $\begin{array}{l}0.000505980374(5)^{a} \\
0.000510671514(3)^{b} \\
0.00050984642212^{c} \\
0.0005266^{d}\end{array}$ \\
\hline 5 & $\begin{array}{l}-22.02943(4)^{a} \\
-22.02890(4)^{b} \\
-22.03097151^{c} \\
\end{array}$ & $\begin{array}{l}-0.03570705(2)^{a} \\
-0.03540033(2)^{b} \\
-0.03540842^{c}\end{array}$ & $\begin{array}{l}-0.00007986(6)^{a} \\
-0.000081905(7)^{b} \\
-0.00007945368^{c}\end{array}$ & $\begin{array}{l}0.02853183(3)^{a} \\
0.02826066(2)^{b} \\
0.0285314619^{c}\end{array}$ & $\begin{array}{l}0.001106259189(7)^{a} \\
0.001108877726(6)^{b} \\
0.001108852957^{c}\end{array}$ \\
\hline 6 & $\begin{array}{l}-32.40465(5)^{a} \\
-32.40389(5)^{b} \\
-32.40624653^{c}\end{array}$ & $\begin{array}{l}-0.07536265(4)^{a} \\
-0.07539549(4)^{b} \\
-0.075395265^{c}\end{array}$ & $\begin{array}{l}-0.000129950(2)^{a} \\
-0.000120606(4)^{b} \\
-0.00012076608^{b}\end{array}$ & $\begin{array}{l}0.06067082(6)^{a} \\
0.06067058(5)^{b} \\
0.06067123554^{c}\end{array}$ & $\begin{array}{l}0.002055698404(1)^{a} \\
0.002064867131(1)^{b} \\
0.0020557067722^{c}\end{array}$ \\
\hline 7 & $\begin{array}{l}-44.77985(6)^{a} \\
-44.77919(6)^{b} \\
-44.78144508^{c}\end{array}$ & $\begin{array}{l}-0.14235340(7)^{a} \\
-0.14230180(8)^{b} \\
-0.142362704^{c}\end{array}$ & $\begin{array}{l}-0.000179550(1)^{a} \\
-0.000177531(4)^{b} \\
-0.00017075579^{d}\end{array}$ & $\begin{array}{l}0.11409910(7)^{a} \\
0.11385820(8)^{b} \\
0.11445597288^{c}\end{array}$ & $\begin{array}{l}0.003426122167(1)^{a} \\
0.003430349716(2)^{b} \\
0.0034302727293^{c}\end{array}$ \\
\hline 8 & $\begin{array}{l}-59.154910(8)^{a} \\
-59.154200(8)^{b} \\
-59.15659501^{c}\end{array}$ & $\begin{array}{l}-0.24635670(1)^{a} \\
-0.24635060(6)^{b} \\
-0.246366726^{c}\end{array}$ & $\begin{array}{l}-0.000229040(1)^{a} \\
-0.000229003(2)^{b} \\
-0.00022939619^{c}\end{array}$ & $\begin{array}{l}0.19745190(1)^{a} \\
0.19794160(1)^{b} \\
0.19794237808^{c}\end{array}$ & $\begin{array}{l}0.005317201324(7)^{a} \\
0.005312586397(8)^{b} \\
0.00531238556282^{d}\end{array}$ \\
\hline 9 & $\begin{array}{l}-75.52969(1)^{a} \\
-75.52895(2)^{b} \\
-75.53171230^{c}\end{array}$ & $\begin{array}{l}-0.39907190(1)^{a} \\
-0.39907480(2)^{b} \\
-0.39906365^{c}\end{array}$ & $\begin{array}{l}-0.000308440(2)^{a} \\
-0.000315806(9)^{b} \\
-0.000296735186^{c}\end{array}$ & $\begin{array}{l}0.32040350(2)^{a} \\
0.32046210(2)^{b} \\
0.32046628674^{c}\end{array}$ & $\begin{array}{l}0.007762084502(3)^{a} \\
0.007782121008(2)^{b} \\
0.0077821042722^{c}\end{array}$ \\
\hline 10 & $\begin{array}{l}-93.904720(1)^{a} \\
-93.904320(3)^{b} \\
-93.90680645^{c} \\
-93.90285(6)\end{array}$ & $\begin{array}{l}-0.61371870(2)^{a} \\
-0.61371590(2)^{b} \\
-0.613704821^{c} \\
-0.613^{d}\end{array}$ & $\begin{array}{l}-0.000372810(8)^{a} \\
-0.000372125(9)^{b} \\
-0.000372719544^{c} \\
-0.000378^{d}\end{array}$ & $\begin{array}{l}0.49023160(2)^{a} \\
0.49133730(2)^{b} \\
0.49264272456^{c} \\
0.491487^{d}\end{array}$ & $\begin{array}{l}0.010919471121(1)^{a} \\
0.010919276981(2)^{b} \\
0.0109192267730^{c} \\
0.011026^{d}\end{array}$ \\
\hline
\end{tabular}

${ }^{a}$ Results obtained using three-parameter wave function $\Psi_{1} .{ }^{b}$ Results obtained using trial wave function $\Psi_{2}$.

${ }^{c}$ Exact results from [15]. ${ }^{d}$ Results obtained using variational Monte Carlo from [16].

TABLE III

The relativistic corrections for the lowest four excited-states of the helium atom. The previous results [19] are cited for the sake of comparison.

\begin{tabular}{|c|c|c|c|c|c|c|}
\hline State & $\begin{array}{c}\text { Nonrelativistic } \\
\text { energies }\end{array}$ & $H_{1}$ & $H_{2}$ & $H_{3}$ & $H_{4}$ & $H_{5}$ \\
\hline $2^{3} S$ & $\begin{array}{l}-2.175005(1) \\
-2.175229376(3)\end{array}$ & $\begin{array}{l}0.000001019(3) \\
0.0000010199(6)\end{array}$ & $\begin{array}{l}-0.000556853(4) \\
-0.0005568(2)\end{array}$ & $\begin{array}{l}-0.000000086(3) \\
-0.000000086(1)\end{array}$ & $\begin{array}{l}0.000441700(3) \\
0.00044186(7)\end{array}$ & 0 \\
\hline $2^{1} S$ & $\begin{array}{l}-2.145971(2) \\
-2.14597351(3) \\
\end{array}$ & $\begin{array}{l}0.000001200(6) \\
0.000001247(1)\end{array}$ & $\begin{array}{l}-0.000547211(4) \\
-0.0005472(3)\end{array}$ & $\begin{array}{l}-0.000000480(1) \\
-0.00000483(2)\end{array}$ & $\begin{array}{l}0.000436605(3) \\
0.0004366(1)\end{array}$ & $\begin{array}{l}0.0000028940(4) \\
0.0000028894(7)\end{array}$ \\
\hline $2^{1} P$ & $\begin{array}{l}-2.123843(1) \\
-2.12384289(7) \\
\end{array}$ & $\begin{array}{l}0.000006435(3) \\
0.000006319(1)\end{array}$ & $\begin{array}{l}-0.000533349(1) \\
-0.0005335(4)\end{array}$ & $\begin{array}{l}-0.00000107(1) \\
-0.000001083(1)\end{array}$ & $\begin{array}{l}0.000426272(3) \\
0.0004262(2) \\
\end{array}$ & $\begin{array}{l}0.0000002450(3) \\
0.0000002448(6)\end{array}$ \\
\hline $2^{3} P$ & $\begin{array}{l}-2.133164(6) \\
-2.13316407(4)\end{array}$ & $\begin{array}{l}-0.000008800(3) \\
-0.000008848(2)\end{array}$ & $\begin{array}{l}-0.000529856(4) \\
-0.0005275(3)\end{array}$ & $\begin{array}{l}0.000001864(2) \\
0.000001864(1)\end{array}$ & $\begin{array}{l}0.000400018(1) \\
0.0004212(1)\end{array}$ & 0 \\
\hline
\end{tabular}

\section{Conclusion}

In this paper we have used variational Monte Carlo method to calculate the relativistic corrections of order $\alpha^{2}$ for the ground state of helium and helium-like atoms and some excited states of helium. Also, the correction due to the nuclear motion was taken into account. The 
obtained results were calculated using two kinds of compact and accurate wave functions. The first one $\left(\Psi_{1}\right)$ is a highly compact wave function that has a clear physical meaning and satisfies all the boundary conditions [31]. The second $\left(\Psi_{2}\right)$ is a simple function having accuracies and shapes similar to those given by Green et al. [33], but with correct cusp conditions [32]. The results in general are much better than those obtained in previous works. Comparing with exact data it is clear that our results are in good agreement with the corresponding exact values. A comparison between results obtained using $\Psi_{1}$ and $\Psi_{2}$ is presented graphically. The comparison shows that the first type $\Psi_{1}$ leads to better results.

For the excited states, the relativistic corrections have been calculated using simple and accurate forms of trial wave functions. The obtained results achieve a good accuracy comparing with previous data. Finally, we conclude that we have used the well known VMC techniques together with new compact and accurate trial wave functions, successfully, to calculate the relativistic corrections for helium isoelectronic and some excited states of helium atom.

\section{References}

[1] S.A. Alexander, R.L. Coldwell, Int. J. Quantum Chem. 63, 1001 (1997).

[2] J.S. Sims, S.A. Hagstrom, Int. J. Quant. Chem. 90, 1600 (2002).

[3] K V. Rodriguez, G. Gasaneo, J. Phys. B, At. Mol. Opt. Phys. 38, 259 (2005).

[4] Y.X. Liu, X.H. Zhang, Y.S. Duan, Mod. Phys. Lett. A 23, 1853 (2008).

[5] H. Nakashima, H. Nakatsuji, J. Chem. Phys. 128, 154107 (2008).

[6] C. Amovilli, N.H. March, I.A. Howard, Á. Nagy, Phys. Lett. A 372, 4053 (2008).

[7] J.S. Sims, S.A. Hagstrom, Int. J. Quant. Chem. 90, 1600 (2002).

[8] V.A. Yerokhin, K. Pachucki, Phys. Rev. A 81, 022507 (2010).

[9] K. Pachucki, J. Phys. B 31, 3547 (1998).

[10] G.W.F. Drake, S.P. Goldman, Can. J. Phys. 77, 835 (1999).

[11] G.W.F. Drake, Can. J. Phys. 80, 1195 (2002).

[12] K. Pachucki, J. Komasa, Phys. Rev. A 68, 042507 (2003).

[13] M. Stanke, D. Kedziera, S. Bubin, L. Adamowicz, J. Chem. Phys. 127, 134107 (2007).
[14] M. Stanke, J. Komasa, D. Kedziera, S. Bubin, L. Adamowicz, Phys. Rev. A 78, 052507 (2008).

[15] C.L. Pekeris, Phys. Rev. 112, 1649 (1958).

[16] S.D. Kenny, G. Rajagopal, R.J. Needs, Phys. Rev. A 51, 1898 (1995).

[17] D. Yi-Shi, L. Yu-Xiao, Z. Li-Jie, Chin. Phys. Lett. 21, 1714 (2004).

[18] M. Stanke, D. Kędziera, S. Bubin, L. Adamowicz, J. Chem. Phys. 126, 194312 (2007).

[19] S.A. Alexander, S. Datta, R.L. Coldwell, Phys. Rev. A 81, 032519 (2010).

[20] V. Korobov, A. Yelkhovsky, Phys. Rev. Lett. 87, 193003 (2001).

[21] K. Pachucki, Phys. Rev. A 74, 022512 (2006).

[22] K. Pachucki, Phys. Rev. A 74, 062510 (2006).

[23] S.B. Doma, F.N. El-Gammal, Appl. Math. Inf. Sci. 5, 315S (2011).

[24] S. Pottorf, A. Puzer, M.Y. Chou, Eur. J. Phys. 20, 205 (1999).

[25] R.W. Hamming, Numerical Methods for Scientists and Engineers, 2nd ed., McGraw-Hill, New York 1973.

[26] A. Papoulis, Probability, Random Variables, and Stochastic Processes, McGraw-Hill, New York 1965.

[27] N. Metropolis, A.W. Rosenbluth, N.M. Rosenbluth, A.M. Teller, E. Teller, J. Chem. Phys. 21, 1087 (1953).

[28] I.I. Sobel'man, Introduction to the Theory of Atomic Spectra, Pergamon Press Ltd, New York 1972.

[29] D. Kedziera, M. Stanke, S. Bubin, M. Barysz, L. Adamowicz, J. Chem. Phys. 125, 084303 (2006).

[30] M.B. Ruiz, Int. J. Quant. Chem. 101, 246 (2005).

[31] D. Bressanini, G. Morosi, J. Phys. B, At. Mol. Opt. Phys. 41, 145001 (2008).

[32] K.V. Rodriguez, G. Gasaneo, D.M. Mitnik, J. Phys. B, At. Mol. Opt. Phys. 40, 3923 (2007).

[33] L.C. Green, M.M. Mulder, P.C. Milner, Phys. Rev. 91, 35 (1953).

[34] S. Chandrasekhar, D. Elbert, G. Herzberg, Phys. Rev. 91, 1172 (1953).

[35] S. Chandrasekhar, G. Herzberg, Phys. Rev. 98, 1050 (1955).

[36] B.H. Bransden, C.J. Joachain, Physics of Atoms and Molecules, Longman Scientific and Technical, London 2003.

[37] T.A. Galek, N.C. Handy, A.J. Cohen, G.K. Chan, Chem. Phys. Lett. 404, 156 (2005).

[38] J. Christophe Pain, M.Sc. Thesis, Ecole Normale Supérieure, Lyon, France, and Indian Institute of Technology, Bombay, India 1997. 